https://idp.uoc.edu

Dossier «Europa ante el reto digital: obstáculos y soluciones»

ARTÍ́CULO

\title{
La «Década Digital» de la Unión Europea: desarrollos e impactos sobre su ciudadanía y economía
}

\author{
David Ramiro Troitiño \\ Profesor titular de la Universidad Tecnológica de Tallin (TalTech)
}

Fecha de presentación: mayo de 2021

Fecha de aceptación: julio de 2021

Fecha de publicación: diciembre de 2021

\begin{abstract}
Resumen
La Unión Europea (UE) es una organización en continua evolución desde su creación a mediados del siglo xx. Su capacidad de adaptación a las necesidades generadas por el proceso de integración es encomiable. La UE ha superado obstáculos imponentes, como la crisis derivada del rechazo a la Comunidad Europea de Defensa y la Unión Política Europea; la activa oposición de un titán político como el presidente francés, De Gaulle; diversas crisis económicas y monetarias; el Brexit y el auge del populismo. Constantemente, la UE ha superado las crisis que le afectaban con la introducción de elementos estabilizadores comunes introducidos por el proceso de integración, creando más Europa. En la actualidad se enfrenta a un nuevo reto, la adaptación a un progreso tecnológico brutal que influirá en el modelo de sociedad imperante en las próximas décadas. Ante este reto, la UE pretende crear un Mercado Único Digital europeo, entre otras iniciativas, que evidentemente tendrá una influencia substancial en las relaciones económicas entre sus miembros y a escala internacional. Esta investigación se centra en el análisis del desarrollo europeo en torno al mundo digital y su influencia en la propia esencia de la organización.
\end{abstract}

\section{Palabras clave}

Europa digital; comercio digital; desarrollo digital de la UE 


\title{
The "Digital decade" of the European Union: developments and impacts on citizens and economy
}

\begin{abstract}
The European Union (EU) is an organisation in continuous evolution since its creation in the middle of the 20th century. Its ability to adapt to the needs generated by the integration process is commendable. The EU has overcome imposing obstacles, such as the crisis derived from the rejection of the European Defence Community and the European Political Union, the active opposition of a political titan such as French President de Gaulle, various economic and monetary crises, the Brexit and the rise of populism. The EU has consistently overcome the crises that affected it by introducing common stabilising elements introduced by the integration process, creating more Europe. The EU is currently facing new challenges, the adaptation to the outstanding technological progress that will influence the prevailing model of society in the coming decades. Faced with this task, the EU wants to create a Digital Single Market for Europe, among other initiatives, which will obviously have a substantial influence on economic relations between its members and also internationally. This research focuses on the analysis of European developments around the digital environment and its influence on the own essence of the organisation.
\end{abstract}

\section{Keywords}

digital Europe; digital trade; digital development of the EU 
1. La digitalización y la automatización como desafíos
para la Unión Europea (UE)

De acuerdo con la definición proporcionada por la Comisión Europea sobre Tecnologías Habilitadoras Clave (THC) en 2009, se incluyen en esta categoría tecnologías vanguardistas tan diversas como la micro y nanoelectrónica, la biotecnología industrial, los materiales avanzados, la fotónica y las tecnologías de fabricación avanzadas. Se estima que actualmente el mercado global de las THC supera los mil millones de euros de facturación, por lo que ya en el presente tiene un impacto significativo en la economía europea.

El desarrollo de nuevas tecnologías, en todas sus vertientes, cambiará radicalmente la vida de los ciudadanos europeos a corto plazo, y la Unión Europea debe adaptarse al cambio (Telefónica, 2020). Por tanto, la organización supranacional europea prioriza la integración digital dentro de un marco social más amplio para un desarrollo equilibrado del proceso de integración europea.

En este sentido, ya se aprecia cómo está cambiando la forma en la que los ciudadanos europeos se comunican, trabajan y usan los servicios de salud, por nombrar algunos ejemplos más notorios. Estos cambios no son solo sociales, sino que también están fuertemente vinculados al desarrollo económico; en los próximos años cambiará la forma en la que se llevan a cabo los negocios y la manera de operar de las empresas. Se espera que se redefina la forma en la que las compañías europeas diseñan, producen, comercializan y generan valor a partir de productos y servicios (Bueno Campos, 2017). La economía digital ya es una realidad, aunque su desarrollo potencial es enorme y se esperan grandes innovaciones en un futuro cercano. Consecuentemente, la Unión Europea debe prestar atención a los progresos de la economía para adecuarse y asumir de manera efectiva un liderazgo digital que estimule la economía europea, fomente el desarrollo y proteja el modo de vida europeo basado en sus valores intrínsecos frente a otros modelos de sociedad altamente digitalizados o en plena transformación, pero con visiones sociopolíticas muy dispares.

El fomento del proceso de digitalización de la Unión Europea crea grandes expectativas de desarrollo econó- mico; se estima que intensificando el proceso se podrían añadir 2500 millones de euros al PIB de la UE para 2025, una cantidad considerable para tan corto periodo de tiempo. Además, teniendo en cuenta la pandemia generada por la COVID-19, el proceso de digitalización económica se ha intensificado de manera natural como respuesta a los problemas de movilidad generados por las restricciones impuestas para frenar la pandemia (Barnes, 2020). Paralelamente, se ha intensificado la necesidad de la Unión Europea, no ya de liderar el proceso, sino de adaptarse a este de la manera más rápida y efectiva, para hacer frente a otros actores internacionales, especialmente Estados Unidos y la República Popular China (Puleri, 2020).

El sector privado no es el único que se ve inmerso en el proceso de digitalización de la sociedad, también se conjetura que el sector público se beneficie enormemente de un uso más amplio de las nuevas tecnologías digitales. Las tecnologías de la información y la comunicación (TIC) permiten que el sector opere de una manera más transparente y eficaz, ya que contribuye a la reducción de la corrupción mediante un control de las actividades públicas más transparente. Asimismo, son relevantes las posibilidades de participación política que ofrece el proceso de digitalización, al permitir que los ciudadanos -incluidas las minorías, los habitantes de las zonas rurales, los discapacitados, o cualquier otro grupo en riesgo de exclusión- tengan acceso digital a la vida política, incrementando así su participación en los asuntos comunes, protegiéndose de situaciones de discriminación y fomentando una visión política más inclusiva (Schou y Hjelholt, 2018). El concepto de participación derivado de la creación de un espacio digital político es también de suma importancia para el avance de la integración desde el punto de vista europeo (Broka, 2015). La Unión Europea es un proyecto de asociación en constante evolución para afrontar las necesidades generadas por el propio proceso. La evolución progresiva de Europa ha alcanzado grandes niveles de integración, pero como en cualquier proceso de crecimiento y profundización, no se ha avanzado en todos los campos de manera homogénea. Desde una perspectiva política, la integración se ha encontrado con obstáculos significativos que han reducido la velocidad del proceso, y que han retrasado este campo en comparación con otras áreas, como la económica (Hanschitz, 2017). El desarrollo digital ayudará a la implementación de un espacio europeo más integrado políticamente, ya que 
reduce las distancias y facilita la participación de todos los ciudadanos europeos en el desarrollo político de la Unión Europea. El futuro político de Europa pasa por involucrar estrechamente a los ciudadanos en su desarrollo, eliminando barreras para que el entramado político común se convierta en una realidad.

El desarrollo digital está imbricado en la optimización de recursos y la reducción de costes tanto a nivel privado como público. Por tanto, la Unión Europea busca acelerar un proceso, ya de por sí inevitable, para mejorar la competitividad económica y el gasto público. Europa se enfrenta a una cuarta Revolución Industrial basada en el desarrollo tecnológico de los últimos años. Las tecnologías avanzadas -como el análisis de datos, la inteligencia artificial (IA), los sistemas automatizados, la computación en la nube, el internet de las cosas (IdC), los robots, la impresión 3D o el 5G-parecen ofrecer infinitas posibilidades de aplicación en casi todos los campos y aspectos de la vida que marcarán el futuro de Europa y del mundo.

Ante esta situación, es necesario analizar el impacto tecnológico en la economía europea y las posibilidades que ofrece al desarrollo del sector público europeo. El carácter supranacional de la Unión Europea y su desarrollo político basado en el concepto jacobino del ciudadano como centro del sistema político, sin tener en cuenta su nacionalidad, género, edad o cualquier otra variable, hace de la organización un embrión para el desarrollo de la ciudadanía digital. Asimismo, el éxito de la creación e implementación del Mercado Único Europeo hace de la adaptación digital una premisa. Ambos temas son desarrollados en este trabajo en busca de una mayor claridad en la actual revolución digital que está transformando el mundo y que, evidentemente, afecta sobremanera a Europa.

\subsection{La Agenda Digital para Europa}

Teniendo en cuenta que la Estrategia de Lisboa, y su revisión introducida en 2005, se agotaron a finales de 2010, la Comisión necesitaba un nuevo marco para afrontar con éxito las mencionadas posibilidades de futuro, por lo que en mayo de 2010 introdujo un nuevo instrumento denominado Agenda Digital para Europa (ADE). Esta, incluía entre sus pilares básicos la adecuación del mercado a la revolución tecnológica a través del Mercado Único Digital. Esta iniciativa busca una economía digital europea sin fronteras en la que se garantice la libre circulación de bienes, personas, servicios y capitales. Este espacio común también promueve la protección efectiva de los datos de los usuarios europeos, ahora en manos de multinacionales que se escapan de los controles de los Estados miembros. En definitiva, se trata de la creación de un área donde los ciudadanos y las empresas puedan acceder sin problemas a bienes y servicios independientemente de la ubicación dentro del espacio económico europeo. La ADE anticipa un mejor uso de las tecnologías de información (TIC) para impulsar el empleo, el crecimiento, la competencia, la inversión y la innovación, y para derribar las barreras que bloquean las oportunidades de un uso más extenso de internet. El $90 \%$ de todas las empresas de la UE son pequeñas y medianas empresas (PYME) y tan solo el $7 \%$ de estas vende en el extranjero. Se espera que la digitalización del Mercado Único cambie esta situación anómala y expanda la actividad económica europea a niveles inimaginables hace tan solo unos años. La Comisión Europea estima que la creación del Mercado Digital Único generará 415.000 millones de euros adicionales a la economía de la UE y el PIB combinado de los Estados miembros crecerá hasta un $1 \%$ anual, y aproximadamente un $2 \%$ en combinación con otros pilares de la ADE. Además, los cálculos de la institución europea indican que se crearán 1,3 millones de nuevos puestos de trabajo para 2025, en un momento de crisis generalizada debido a la pandemia que ha afectado enormemente al mercado laboral europeo (Parlamento Europeo, 2019). Un mercado único verdaderamente digital generará nuevas oportunidades comerciales, promoverá la innovación y fomentará la creación de nuevas empresas. Esta visión idílica se enfrenta a numerosos obstáculos, entre los que destaca el nacionalismo económico, el poder de las grandes empresas tecnológicas, la falta de un marco común en materia impositiva, o la imposición de medidas de efecto equivalente a aranceles por parte de los Estados miembros para proteger sus intereses particulares. La hercúlea tarea de sobrepasar estas adversidades dependerá enormemente de la situación económica de Europa, donde un crecimiento robusto y continuado comparado con otros actores internacionales será un factor fundamental. La receta para el éxito es voluntad política para avanzar en la integración digital de la económica, reduciendo sus efectos adversos sobre los agentes económicos y los consumidores. Pero dicha voluntad puede ser crucialmente influenciada por la situación internacional. Si Estados Unidos o la República Popular China avanzan más que Europa, los gobernantes entenderán que la integración digital es una necesidad y 
sacrificarán sus intereses más particulares en aras de un desarrollo común europeo. Sin embargo, si Europa consigue afianzarse en la economía digital global de manera efectiva, sin comunalizar demasiado, estos no tendrán el estímulo necesario para implementar una reforma tan relevante y que tendrá un impacto considerable en la economía europea.

La hoja de ruta definida por la Comisión para alcanzar el Mercado Digital Europeo está basada en tres pilares fundamentales sobre los cuales el entramado digital europeo descansará en un futuro cercano. El primer pilar, vinculado con el acceso digital, consta de elementos tan destacados como el comercio en internet, la distribución y logística de productos encargados a través de la red, la eliminación del geobloqueo en todo el territorio europeo, leyes de copyright que protejan a los creadores y fomenten la innovación mediante de la creación de un marco regulatorio a nivel europeo y con influencia mundial, y un tipo de impuesto de valor añadido estándar para toda las compañías que trabajan en el Mercado Digital Europeo para evitar distorsiones que afecten a la competitividad $y$, así, eliminar barreras fiscales que afecten a la igualdad de condiciones para todos los operadores europeos.

El siguiente desarrollo pasa por la creación de un entramado estable que apoye los desarrollos tecnológicos necesarios para la implementación del Mercado Único Digital. El desarrollo de un marco regulatorio común para las grandes empresas tecnológicas de telecomunicación y un marco común para los medios de información digital son objetivos prioritarios para la Unión Europea, ya que se espera que faciliten un espacio estable en el cual el desarrollo europeo pueda asentarse. En este mismo capítulo se incluyen acciones para el fomento y la creación de plataformas online a escala europea y aspectos concernientes a la seguridad y los datos personales. Europa busca proteger a sus ciudadanos de los efectos perniciosos del desarrollo digital con la creación de una legislación protectora con vocación previsora más que correctora. Anticiparse a los problemas dará más credibilidad al esfuerzo digital de la UE.

El tercer pilar se basa en aspectos relacionados con la economía y la sociedad, e incluye aspectos importantes diversos, como el mercado de datos, uno de los principales campos de desarrollo, tanto para la venta como para la producción y la implementación de tecnologías basadas en algoritmos que necesitan un gran flujo de datos para su correcto funcionamiento, como la inteligencia artificial. Regular el acceso y la distribución de dichos datos es fundamental para el desarrollo económico sostenible en un Mercado Único Digital. Otros aspectos básicos, son la introducción de estándares europeos. Un mercado requiere de normas comunes para su correcto funcionamiento, de manera que se eviten competencias desleales, discriminaciones y alteraciones artificiales del funcionamiento basado en la libre competencia, donde los más competitivos sobreviven y los demás desaparecen (Lips, 2020). La selección natural explicada por Darwin puede perfectamente aplicarse al desarrollo tecnológico empresarial, aunque la delicada etapa inicial necesita de cierta protección y dirección para evolucionar de manera acorde con los principios sociales europeos. Desde el punto de vista político, cabe destacar el esfuerzo realizado para el desarrollo del Gobierno digital o gobernanza electrónica, que es la aplicación de tecnología para la prestación de servicios gubernamentales, el intercambio de información, las transacciones de comunicación, la integración de varios sistemas independientes entre el Gobierno y el ciudadano, la dirección empresarial, la gestión laboral, así como procesos e interacciones de la parte administrativa dentro de todo el marco gubernamental. Mediante la gobernanza electrónica, los servicios de la Unión Europea se pondrían a disposición de los ciudadanos de una manera conveniente, eficiente y transparente (Kerikmäe et al., 2019). Los tres principales grupos destinatarios se pueden distinguir en tres niveles diferentes: el administrativo e institucional a nivel europeo, los ciudadanos y las empresas y los grupos de interés.

Una vez establecidos los cimientos, su desarrollo hará posible la implantación de un Mercado Digital europeo que fortalezca el proceso de construcción europea internamente, al crear reglas comunes y un espacio digital sin fronteras entre los Estados miembros. A su vez, exteriormente, una Europa digital fuerte protegerá el modo de vida de esta y de sus ciudadanos frente a gigantes tecnológicos extranjeros cuyos parámetros de desarrollo son discordantes con la visión europea de sociedad.

\subsection{Nuevos retos}

La Unión Europea ha realizado grandes avances adecuándose a las circunstancias socioeconómicas derivadas de la revolución tecnológica; alguna de sus iniciativas se encuentra en los albores con enormes posibilidades de 
desarrollo, otras han fracasado y algunas han sido eficientemente implementadas. El Mercado Único Digital presenta incontables oportunidades, pero también se enfrenta a retos significativos que pueden lastrar su desarrollo y anclar el progreso europeo frente a otras zonas del mundo. En primer lugar, hacer que el Mercado Único de la Unión Europea se adapte a la era digital requiere derribar barreras reglamentarias y pasar de los mercados nacionales individuales a un único código normativo para toda la UE. La creación de un marco legal común a nivel europeo se enfrenta a la oposición del nacionalismo político, que aboga por una autonomía estatal frente a los impulsos uniformadores que emanan desde Bruselas. El concepto de la soberanía nacional desempeña un papel relevante en el proceso de construcción europea desde sus inicios hasta la actualidad, desarrollando un juego de equilibrios entre la cooperación y la integración, entre el intergubernamentalismo y el federalismo, entre una comunidad de Estados independientes y la creación de un espacio común (Iwabuchi, 2019). Por tanto, la Unión Europea, actualmente muy integrada, ha evolucionado constantemente desde la cooperación hacia la integración, en un proceso difícilmente reversible, pese a las constantes tensiones que, por ejemplo, han derivado en la salida del Reino Unido de la UE. El Brexit puede suponer el espaldarazo definitivo para la creación de un marco legislativo común en la Europa digital, ya que el país anglosajón era el adalid europeo de la cooperación, mostrando constantemente su reticencia a aumentar los niveles de integración a nivel europeo (Troitiño et al., 2018).

Otro hecho fundamental para el desarrollo de un acervo común digital es la incapacidad de los Estados para influir en el mundo digital, al no poder controlar las grandes corporaciones tecnológicas que dominan el mundo digital, de manera que imponen sus propias leyes sin cortapisas nacionales incapaces de dictaminar normas de control efectivas. Solo la unión del esfuerzo regulador común daría como resultado un espacio suficientemente relevante para acotar las acciones de los gigantes de internet dentro de un marco estable que respete el modelo de sociedad europeo. Por tanto, el debate sobre la soberanía nacional en temas digitales es fútil, porque la única manera de ejercer cierta soberanía de manera efectiva es mediante la colaboración europea y que las fronteras del mundo digital sobrepasen las de los Estados europeos. Tan solo gigantes como los Estados Unidos o China han mostrado músculo suficiente para ejercer un control efectivo sobre el mundo digital, aunque partiendo desde paradigmas radicalmente dispares y con resultados divergentes (Liaropoulos, 2017).

Existen numerosas iniciativas (a menudo descoordinadas) encaminadas a una estrategia legal clara tanto a nivel de la UE como de los Estados miembros. La necesidad de presentar una estrategia común, respaldada por todos los agentes relevantes involucrados, es un reto que requiere una coordinación profunda. El mundo digital está en sus inicios, por tanto, cuanto más avance la legislación a escala nacional, mayores serán las divergencias a nivel europeo y más difícil será la integración futura. Ante este hecho, la Comisión Europea pretende avanzar en la integración antes de que la proliferación de obstáculos nacionales ralentice el proceso de manera significativa en el futuro, rezagando Europa a nivel mundial en un sector clave para la economía y la sociedad. Estos pasos podrían contribuir con 415.000 millones de euros al año al crecimiento económico europeo, lo que impulsa el empleo, la competencia, la inversión y la innovación en la UE (Giovinazzi, 2020), asienta la influencia internacional de Europa y protege a los europeos de gigantes mundiales dirigidos por Gobiernos totalitarios o que simplemente se guían por la maximización de beneficios sin cortapisas sociales y con contribuciones básicas al desarrollo de las sociedades donde operan.

La nueva Europa digital necesita de un marco legal donde asentarse y expandirse de manera efectiva y respetuosa con los principios que guían a la sociedad europea. Por tanto, la Unión Europea ha trabajado en este sentido elaborando un primer informe anual sobre la situación del Estado de derecho en la Unión Europea 2020, en el que se incluyen cuestiones digitales. El concepto general del marco europeo enfatiza el desarrollo de la resiliencia económica y del mercado laboral con sostenibilidad económica, social, ambiental e institucional como principio rector de las políticas europeas al respecto. Se espera que este acercamiento fomente la convergencia ascendente y la equidad en la transición hacia una economía climáticamente neutra, al tiempo que se gestionan los desafíos que plantean la digitalización y el cambio demográfico (Comisión Europea, 2020). Dada la rápida evolución de este campo, la UE prevé requisitos adicionales para revisar los desarrollos en los Estados miembros. 


\section{Beneficios para el sector público europeo}

Pese a que las posibilidades económicas son las más plausibles dentro de una revolución tecnológica que crea mercados globalizados, donde las barreras entre productores y consumidores no están afectadas por las fronteras tradicionales, existen otros campos en los cuales la Unión Europea espera unos réditos considerables. Ante el debate sobre el carácter de la Unión Europea, económico o político, la realidad proporciona una respuesta híbrida. Por tanto, los avances tecnológicos y la implementación del Mercado Único Digital tienen también ventajas más allá del plano económico, ya de por sí considerable, enfocadas hacia el servicio público de los ciudadanos. La Unión Europea está ante una gran oportunidad de mejorar la vida de sus ciudadanos y, por tanto, de incrementar el apoyo popular al proyecto de construcción europea. La digitalización tiene un gran potencial para el desarrollo político de la institución y para acelerar el proceso de integración de manera considerable. Existen campos estratégicos cuyo poder de arrastre implica otras políticas y, por tanto, son una prioridad para la UE.

\subsection{Democracia}

Desde el comienzo del proceso de integración, la UE se ha enfrentado al problema de la capacidad de una entidad supranacional de ejercer de pleno derecho las prerrogativas de una entidad soberana común. Uno de los constantes obstáculos para la integración ha sido la reticencia de ciertos Estados y agentes sociales a permitir que la soberanía pudiera ser ejercida más allá de lo estatal, que en numerosos casos es a su vez nacional por la imbricación de dos conceptos diferenciados, Estado y nación. Los defensores de esta postura abogan por una cesión mínima de soberanía a una Europa vinculada orgánicamente con el concepto de cooperación. Pero en cierta manera se apropian de la soberanía, que en realidad reside en los ciudadanos de sus Estados (no en sus nacionales, ni en el propio Estado). Los sujetos políticos son quienes, de acuerdo con un contrato social, ceden la gestión de su libertad personal al entramado político para la creación de reglas comunes que permitan la convivencia armónica en sociedad de una amalgama variada de individuos. La aceptación de dichas reglas por parte de los integrantes del sistema, los ciudadanos, es lo que se conoce como lealtad institucional desde un punto de vista político, o patriotismo desde un punto de vista político-cultural. El conjunto de los derechos cedidos por parte de los ciudadanos (sujetos políticos) conforma la soberanía. El Estado no es necesariamente el último receptor de esta cesión ni es el dueño de la soberanía, sino su gestor público. El problema de la soberanía europea puede ser solventado, o mitigado, con la implementación de soluciones digitales para los problemas de los ciudadanos, atrayendo su lealtad al proyecto europeo, al ser la UE más efectiva a la hora de solucionar los problemas ciudadanos (Jabko y Luhman, 2019). Las soluciones digitales pueden incrementar el apoyo a la UE entre los europeos que aun hoy en día afrontan de manera confusa la creación de una casa común por el carácter dual de su lealtad institucional, por un lado, dominada por la racionalidad basada en la gestión eficaz de los problemas que les atañen y, por otro, predeterminada por las emociones emanantes del concepto de nación que se confunde con política debido al fomento del Estado-nación a partir del siglo xıx para atraer de una manera más efectiva la lealtad de los miembros de la sociedad (Hamul'ák, 2016). La UE no puede competir con las emociones, pero sí con la racionalidad. Si se muestra efectiva en la solución de los problemas de los ciudadanos, atraerá suficiente lealtad o apoyo para avanzar en el proceso de integración. Asimismo, incrementar la participación cívica en los asuntos europeos mediante el fomento de herramientas digitales, influirá enormemente en la creación de la casa de todos los europeos.

\subsection{Impacto social de la integración digital económica}

El Mercado Único Digital permitiría a la UE la gestión de servicios online conectando de forma armónica los sistemas de información electrónicos del sector público de los Estados miembros y de las propias instituciones europeas, coordinando la gestión, evitando congestiones, diseñando mejores trazados digitales y optimizando las respuestas ante situaciones de emergencia. Paralelamente, la libre circulación de personas y mercancías actuales dentro de la Unión Europea ha creado sistemas viales que transcienden las fronteras estatales y que necesitan una gestión común para su correcto funcionamiento. La digitalización de la gestión permitiría grandes avances y numerosos beneficios para los ciudadanos como modelos para predecir problemas con la calidad de la carretera, accidentes de tráfico, problemas de velocidad, rentabilidad, etc. (Mihet-Popa y Saponara, 2018). 
El impacto del Mercado Único Digital depende enormemente de la capacidad de generar datos suficientes para la creación de modelos efectivos. La dependencia de datos fiables es enorme y fundamental, lo que coloca a Europa por delante de otros gigantes mundiales, gracias al avance del sector europeo en comparación con competidores internacionales. Esta ventaja es temporal, ya que la generación de datos es una prioridad global y las inversiones son significativas a escala planetaria, constatando la máxima de que la información es poder. La Unión Europea tiene como prioridad invertir en la generación de datos por su potencial y por la necesidad inherente de estos. La idea es simple: la generación de datos es más rentable y efectiva a gran escala, por lo que parece adecuado que sea la UE quien se encargue de este aspecto crucial para el futuro más que los Estados miembros, capaces de generar bases de datos menores. Como ejemplo ilustrativo, vemos cómo la Política Agraria Común, dependiente de la Unión Europea, necesita de una gran cantidad de datos para incrementar su efectividad y proteger el medio rural europeo en todas sus dimensiones. El análisis para determinar el estado y la calidad de la tierra utilizada en labores agrícolas y ganaderas se realiza más efectivamente por satélite, por lo que sería más sabio emplear un satélite único europeo a que cada miembro utilice su propio satélite. Le generación de datos también es indispensable para una gestión eficaz del sistema sanitario y la posibilidad de analizar las necesidades médicas de un paciente y donde pueden ser mejor atendidas de acuerdo con los recursos disponibles. La implementación de un sistema digital de coordinación sanitario será de máxima importancia para la Unión Europea por su claro beneficio para los ciudadanos, la mejora de su salud, la optimización de recursos y la planificación racional (Padrón, 2018). Aunque los obstáculos son numerosos en este tema, debido a la fuerte identificación de los países con su sistema sanitario como parte transcendente de su identidad común, de su imaginario colectivo. En cualquier caso, todos estos ejemplos apuntan en la misma dirección: Europa afronta grandes retos en términos de integración digital, pero ofrece grandes posibilidades para avanzar positivamente en el proceso de integración por los beneficios potenciales que puede ofrecer a sus ciudadanos.

La economía digital es un fenómeno nuevo y aún desconocido que se refleja en el crecimiento exponencial de las plataformas en línea que utilizan trabajadores que no tienen un empleo a tiempo completo y que tienen horarios flexibles. Parte de ellas son plataformas de economía colaborativa que se especializan en tareas puramente digitales que no requieren presencia física o proximidad entre trabajadores y sus clientes - mercados laborales en línea-. Todos los sectores -desde especialistas en la tecnología de la información ( $\mathrm{TI}$ ) hasta taxistas o repartidores de alimentos, incluyendo todos los grupos de edad- muestran una tendencia creciente y transfronteriza. Este carácter supraestatal de la economía digital crea numerosos problemas de control por parte de las autoridades de los Estados individuales, una disfunción que puede ser resuelta con una gestión común. Por tanto, la gestión en Europa no conlleva un socavamiento de la soberanía de los Estados miembros, ya que estos carecen del poder necesario para hacer efectiva dicha soberanía en el contexto de la economía digital (Joamets y Chochia, 2020). El liderazgo europeo en el control y la regulación de la economía digital no es solamente recomendable, sino una necesidad básica para el correcto funcionamiento del sistema político y social de sus miembros.

La situación laboral de los trabajadores de la economía digital en la UE/EEA ha sido analizada por la Comisión Europea, y ha arrojado datos que refuerzan la necesidad de una acción común. El estudio se ha centrado en las restricciones aplicables a las aplicaciones de transporte compartido y entrega de comida, lo que ha revelado la gran cantidad de factores de impacto y partes interesadas. Mientras que algunos países europeos favorecen regulaciones laborales estrictas, otros intentan definir «una tercera categoría de trabajadores» o luchan por atraer innovaciones. En países como Alemania, Austria, Dinamarca, Finlandia, Francia Suecia y Suiza, los empleados de la economía digital se consideran empleados de la empresa con todas las consecuencias legales. Esto implica el pago de impuestos sociales, el control del Estado sobre las relaciones laborales que se tienen que adecuar a la legislación pertinente sin existir una tercera categoría de trabajadores. Asimismo, en este grupo de países existe una fuerte presión por parte de los sindicatos para regular estas relaciones laborables. Un segundo grupo de países incluye el Benelux, España, Hungría, Italia, IsIandia, Noruega, Polonia, Portugal y la República Checa, donde se han introducido ciertas restricciones a la economía digital en materia laboral, con una tendencia hacia la creación de estándares comunes. Aunque se esperan resoluciones judiciales futuras sobre la gestión laboral de la economía digital, por los numerosos casos presentados 
ante los tribunales nacionales. Existe una emergente tercera categoría de trabajadores que cuentan con ciertos derechos laborables, por ejemplo, un número máximo de horas o un seguro sanitario, aunque todavía existe un debate político-social al respecto. Por último, existe un tercer grupo de países, que incluye a Bulgaria, Chipre, Croacia, Eslovaquia, Estonia, Grecia, Letonia, Lituania y Rumanía, sin una legislación suficientemente definida sobre el estatus de los trabajadores de la economía digital, pudiéndose equiparar con la figura de trabajadores autónomos, por lo que los pagos de impuestos sociales no conciernen a la plataforma digital que emplea a los trabajadores (Berg et al., 2018).

Estas divergencias en el incipiente mercado digital están creando una alteración del actual Mercado Único Europeo, porque pese a que haya reglas laborables especificas en cada país y no sea una prerrogativa europea legislar al respecto, vemos cómo estas plataformas funcionan en Europa, lo que produce una distorsión en el sistema que puede alterar de manera importante la competitividad de las economías de los países miembros. Una armonización total europea en este campo es hartamente improbable por los vínculos de la política laboral con el sistema social. Sin embargo, la disfunción actual puede ser resuelta con la introducción de una postura general común ante el reto planteado por la economía digital, que solo puede ser implementado con la creación de un Mercado Único Digital. Los estándares comunes servirán para que las divergencias entre las distintas economías se reduzcan y potencien la convergencia a escala europea, pero permitiendo diferencias significativas según las necesidades y los modelos imperantes en cada país.

\section{La digitalización de las instituciones democráticas europeas}

El Parlamento Europeo es una de las instituciones centrales de la Unión Europea. Su evolución, en términos de relevancia en el entramado institucional de esta, es constante. Partiendo de una mera asamblea consultativa a inicios del proceso de integración europea, ha evolucionado hacia un parlamento democrático que representa a los ciudadanos de la Unión Europea y participa activamente en la aprobación de leyes. No obstante, el proceso evolutivo del
Parlamento presenta diversos problemas que podrían ser fácilmente resueltos desde una perspectiva digital.

En sus inicios, el Parlamento Europeo (PE) estaba localizado en Luxemburgo, pero la negativa del Gran Ducado a acoger más instituciones, pero sin perder las ya adquiridas, derivó en la creación de múltiples sedes para aquel. Francia acogió la sede de las reuniones plenarias en Estrasburgo, ciudad símbolo de la paz entre Alemania y Francia, ya que fue foco de conflicto entre ambas entidades en el pasado. A su vez, las oficinas principales del PE se situaron en Bruselas, centro neurálgico de la Unión Europea, por motivos meramente logísticos. Más allá de las lógicas nacionalistas, y en cierta manera obsoletas, la sede del Parlamento Europeo debería contar con un único emplazamiento, Bruselas, el centro de la toma de decisiones real de la Unión. En la actualidad, los servicios del PE se dividen en tres ciudades, Bruselas, Estrasburgo y Luxemburgo. La digitalización de los servicios y la burocracia parlamentaria reduciría estas localizaciones a dos, y reduciría enormemente los traslados de los parlamentarios y sus equipos y el transporte de toneladas de informes entre estas tres localizaciones. El traslado desde Estrasburgo a Bruselas, para conseguir la sede única, es un problema político en el que la digitalización solo puede mitigar sus efectos perniciosos, pero no solventarlo.

En lo referente a las elecciones al Parlamento Europeo, en la actualidad se rigen de acuerdo con las normas estatales pertinentes, lo que crea divergencias considerables en el sistema. Por ejemplo, en lo que respecta a la edad de votación, que es de dieciséis en Austria y se fija en los dieciocho en el resto de los países miembros, se producen diferencias entre los ciudadanos europeos dentro de un marco político común. Existen algunas reglas europeas, pero las diferencias son todavía considerables y reducen la eficiencia del sistema a la hora de generar un demos europeo, un cuerpo elector europeo. Si el Parlamento Europeo fuera elegido por los ciudadanos europeos, ya que representa a los ciudadanos de Europa, se debería contar con un procedimiento común en sus elecciones. La implementación de un sistema electoral único basado en un sistema de votación digital (siguiendo el ejemplo de países como Estonia) subsanaría este problema y fomentaría la, hasta favorecer la igualdad entre todos los ciudadanos europeos. Los principales obstáculos nacen de la negativa de los propios Estados miembros a abandonar sus tradiciones electorales en aras de un bien común (de solución 
política), y de la barrera a la que se enfrentarían aquellos ciudadanos no familiarizados con el mundo digital. Por tanto, un acercamiento híbrido, en el que se mantiene el voto presencial y se introduce el voto digital, supondría una transición moderada hacia un modelo más sostenible desde el punto de vista de participación ciudadana.

Otro de los obstáculos relevantes para la implementación de una democracia más efectiva en Europa está relacionado con la carencia de partidos políticos a escala europea. Las elecciones europeas están dominadas por los partidos políticos nacionales, por tanto, la discusión durante la campaña está más centrada en los problemas de ámbito estatal que en los problemas y las prioridades europeas. Teniendo en cuenta que los Estados miembros ya se encuentran representados en el Consejo, el Parlamento Europeo debería trabajar de acuerdo con los intereses europeos, obviando las necesidades particulares de cada Estado. Por lo tanto, son necesarios los partidos políticos europeos para priorizar las acciones a escala europea. Como solución, se podría implementar un sistema donde solo los partidos presentes en al menos el $40 \%$ del territorio de la Unión, o en áreas donde más del $40 \%$ de la población europea resida, podrían presentarse a las elecciones europeas. Esto obligaría a la creación de partidos políticos europeos sobre la base de los nacionales, pero su perspectiva sería mucho más europea, ya que se centrarían en las cuestiones comunes a la Unión Europea, y más independiente de los problemas particulares de cada miembro. Las dificultades geográficas para implementar este sistema serian fácilmente evitables con la creación de plataformas digitales políticas europeas. De igual manera, la barrera lingüística podría ser evitada con la creación de páginas webs en distintos idiomas de una manera sencilla. La creación de eventos en línea solventaría el problema de las distancias. Es evidente que un acercamiento digital facilitaría la creación de entidades políticas en Europa, aunque con el riesgo de abandonar a aquellos sin las capacidades ni el interés necesarios para participar digitalmente. Por tanto, el nivel de educación y capacitación de los europeos en lo digital debe ser potenciado desde la Unión Europea antes de implementar un paso tan importante y con consecuencias políticas fundamentales para los ciudadanos europeos.

En relación con la participación ciudadana, un tema que puede generar grandes sinergias a nivel europeo, es la convocatoria de referéndums europeos sobre temas de la
UE bajo el control del Parlamento europeo. Es extraño que muchas medidas no se discutan en Europa cuando tienen una influencia determinante sobre los ciudadanos de la Unión Europea. Si el Parlamento Europeo pudiera organizar referéndums europeos, la gente sentiría la presencia de esta institución mucho más, y que su opinión está representada. Dado que la UE no es un Estado nacional, los referéndums europeos deberían ser más restrictivos y respetar el equilibrio de poder de las instituciones europeas, y por tanto la aprobación del Consejo debería ser obligatoria. La logística es otro de los factores que influyen negativamente en el desarrollo de referéndums en Europa, algo fácilmente salvable con la estructura digital adecuada. La Europa digital daría voz a los ciudadanos, aunque sin caer en los excesos de la democracia ateniense, sino respetando los límites legales y el equilibrio institucional.

Por último, la revolución digital está en condiciones de contribuir significativamente a la reducción de la corrupción y la mala administración en el Parlamento Europeo. La reforma digital de este supondría una mayor claridad y un menor índice de mala praxis. A mayor claridad y acceso a la información por parte de los ciudadanos, menor es el riesgo de corrupción entre los representantes políticos de los europeos, ya que sus acciones serían más públicas y se reduciría el margen de maniobras opacas.

Tras lo aquí expuesto, parece razonable que el Parlamento Europeo acometa una transformación digital profunda para afrontar de manera eficiente los retos del siglo XXI y cumplir así con las expectativas generadas por el proceso de construcción europea en su vertiente más política (Floridi, 2020).

En lo referente al desarrollo normativo de la Europa digital, el PE tiene una gran influencia debido a su peso en el proceso legislativo europeo. El procedimiento de codecisión se introdujo por primera vez en 1992 y su uso se amplió en 1999. Con la adopción del Tratado de Lisboa, la codecisión pasó a denominarse procedimiento legislativo ordinario y se convirtió en el principal procedimiento de toma de decisiones para la adopción de la legislación de la UE. Se aplica en unos 85 ámbitos de actuación y la responsabilidad final recae en el PE y en el Consejo de la UE. Si una propuesta legislativa es rechazada en cualquiera de las fases del procedimiento o si el Parlamento Europeo y el Consejo no pueden llegar a una solución transaccional, 
la propuesta no se adopta y se da por concluido el procedimiento (Frosio, 2017). La revolución digital supondría aligerar el proceso, comúnmente atacado por su falta de agilidad, e intensificar las relaciones del PE y el Consejo de manera digital.

\section{Conclusiones}

La Unión Europea afronta un reto sin precedentes en términos de evolución marcado por el desarrollo tecnológico. La organización avanza, aunque se enfrente a poderosos obstáculos que amenazan un desarrollo y una ejecución conjuntos que maximizarían las acciones comunes y dotarían a la organización de relevancia internacional, bienestar económico e independencia interna.

La UE tiene una sólida base científica e industrial sobre la que construir, con laboratorios de investigación líderes y universidades destacadas mundialmente, así como numerosas empresas innovadoras. Tiene un marco legal integral que protege a los consumidores al tiempo que promueve la innovación y está avanzando en la creación de un Mercado Único Digital. Los ingredientes principales están ahí para que la UE se convierta en un líder en la revolución tecnológica mundial, a su manera y en función de sus valores. El enfoque digital descrito en este capítulo muestra el camino que Europa quiere seguir y la necesidad de unir fuerzas a nivel europeo para garantizar la transformación digital homogénea, sin vencedores ni perdidos, dedicándose recursos adecuados al Mercado Digital único y su culminación. Es importante resaltar el compromiso de la UE en el desarrollo digital y su convergencia con los valores de la Unión y los derechos fundamentales. La Unión Europea cuenta con los instrumentos para convertirse en un líder mundial de un nuevo equilibrio internacional, donde las nuevas tecnologías desempeñarán un papel relevante. La incapacidad del nacionalismo de compartir más soberanía nacional en Europa puede ser contrarrestada por la complejidad del mundo digital, que alejará del debate populista la conveniencia de un manejo común. Aunque la lógica no lo es todo en las relaciones sociales y la UE tiene que mantenerse firme en su estrategia actual.

La conectividad internacional del Mercado Digital Único es una premisa básica para su maximización y es ahí donde las relaciones digitales con América Latina son fundamentales. Para fomentar esa conexión, la UE debe fomentar la interdisciplinariedad en proyectos e innovación (medicina online, inteligencia artificial en el mercado laboral) con los socios iberoamericanos, apoyar las capacitaciones transfronterizas sobre GDPR y GovTech, facilitar la publicación conjunta en revistas altamente indexadas e invitarse mutuamente a foros (online) y grupos de expertos para un mayor desarrollo de las relaciones digitales entre ambas áreas. 


\section{Referencias bibliográficas}

BARNES, S. J. (2020). «Information management research and practice in the post-COVID-19 world». En: International Journal of Information Management, núm. 55, págs. 102-175 [en línea]. DOI: https://doi.org/10.1016/j.ijinfomgt.2020.102175

BERG, J.; FURRER, M.; HARMON, E.; RANI, U.; SILBERMAN, M. S. (2018). Digital labour platforms and the future of work: Towards decent work in the online world. Ginebra: International Labour Office.

BROKA, S. (2015). Nākotnes perspektīvas suverēnās labklājības fondu darbībā Persijas Līča Sadarbības padomes reǵionā.

BUENO CAMPOS, E. (2017). El análisis organizativo de la empresa en la economía digital.

COMISION EUROPEA (2020). «Informe anual sobre la situación del Estado de derecho en la Unión Europea» [en línea]. Disponible en: https://ec.europa.eu/info/policies/justice-and-fundamental-rights/ upholding-rule-law/rule-law/rule-law-mechanism/2020-rule-law-report_es [Fecha de consulta: 12 de enero de 2021].

CHRISTOU, G. (2019). «The collective securitisation of cyberspace in the European Union». En: West European Politics, vol. 42, núm. 2, págs. 278-301 [en línea]. DOI: https://doi.org/10.1080/01402382 .2018.1510195

FLORIDI, L. (2020). «The fight for digital sovereignty: What it is, and why it matters, especially for the EU». En: Philosophy \& Technology, vol. 33, núm. 3, págs. 369-378 [en línea]. DOI: https://doi. org/10.1007/s13347-020-00423-6

FROSIO, G. F. (2017). «Reforming intermediary liability in the platform economy: a European digital single market strategy». En: Nw. UL Rev. Online, vol. 112, núm. 18 [en línea]. DOI: https://doi.org/10.2139/ ssrn.3009155

FUNDACIÓN TELEFÓNICA (2020). Sociedad Digital en España 2019. Fundación Telefónica.

GIBADŁO, L. (2020). «Coronapresidency»: German Priorities for the Presidency of the Council of the EU.

GIOVINAZZI, E. (2020). European Union fit for the digital age: a transformation that benefits all citizens.

HAMUL'ÁK, O. (2016). National sovereignty in the European Union: View from the Czech perspective [en línea]. Springer. DOI: https://doi.org/10.1007/978-3-319-45351-4

HANSCHITZ, G. (2017). Digitalization of Politics and Elections [en línea]. DOI: https://doi.org/10.1007/9783-319-06091-0_3-1

HOFFMANN, T., \& PRAUSE, G. (2018). «On the regulatory framework for last-mile delivery robots». En: Machines, vol. 6, núm. 3, pág. 33 [en línea]. DOl: https://doi.org/10.3390/machines6030033

IWABUCHI, K. (2019). «Globalization, Digitalization, and Renationalization: Some Reflections from Japanese Cases». En: Situations, vol. 12, núm. 1, págs. 1-22.

JABKO, N.; LUHMAN, M. (2019). «Reconfiguring sovereignty: crisis, politicization, and European integration». En: Journal of European Public Policy, vol. 26, núm. 7, págs. 1037-1055 [en línea]. DOI: https://doi.org/10.1080/13501763.2019.1619190

JOAMETS, K.; CHOCHIA, A. (2020). «Artificial Intelligence and Its Impact on Labour Relations in Estonia». En: Slovak Journal of Political Sciences, vol. 20, núm. 2, págs. 255-277 [en línea]. DOl: https:// doi.org/10.34135/sjps.200204

KASPER, A.; VERNYGORA, V. A. (2020). Towards a 'cyber Maastricht': Two steps forward, one step back. 
KERIKMÄE, T.; TROITIÑO, D. R.; SHUMILO, O. (2019). «An idol or an ideal? A case study of Estonian e-Governance: Public perceptions, myths and misbeliefs». En: Acta Baltica Historiae et Philosophiae scientiarum, vol. 7, núm. 1, págs. 71-80 [en línea]. DOI: https://doi.org/10.11590/abhps.2019.1.05

LIAROPOULOS, A. N. (2017). «Cyberspace governance and state sovereignty». En: Democracy and an Open-Economy World Order, págs. 25-35 [en línea]. Springer, Cham. DOI: https://doi.org/10.1007/9783-319-52168-8_2

LIPS, W. (2020). «The EU Commission's digital tax proposals and its cross-platform impact in the EU and the OECD». En: Journal of European Integration, vol. 42, núm. 7, págs. 975-990 [en línea]. DOI: https://doi.org/10.1080/07036337.2019.1705800

MARTÍN DE LA GUARDIA, R.; PÉREZ SÁNCHEZ, G. (2001). Historia de la integración europea. Barcelona: Ariel.

MARTÍNEZ RAMÍL, P. (2017). Donald Trump, un análisis comparativo del discurso populista en EE. UU.

MAZZUCATO, M. (2018). Mission-oriented research \& innovation in the European Union.

MIHET-POPA, L.; SAPONARA, S. (2018). «Toward green vehicles digitalization for the next generation of connected and electrified transport systems». En: Energies, vol. 11, núm. 11, pág. 3124 [en línea]. DOI: https://doi.org/10.3390/en11113124

OUTEDA, C. C. (2001). El proceso de constitucionalización de la Unión Europea: de Roma a Niza, núm. 3. Santiago de Compostela: Universidad Santiago de Compostela.

PADRÓN, N. A. (2018). «De los algoritmos a la salud. La inteligencia artificial en la atención sanitaria». En: Revista de Occidente, núm. 446, págs. 63-75.

PARLAMENTO EUROPEO (2019). «Una agenda digital para Europa». Fichas Temáticas sobre la Unión Europa [en línea]. Disponible en: http://www.europarl.europa.eu/factsheets/es/sheet/64/una-agenda-digital-para-europa [Fecha de consulta: 12 de enero de 2021].

PULERI, M. (2020). «Engaging with European (Dis-)Integration: Russia in Dialogue with Europe/s». En: Rekindling the Strong State in Russia and China, págs. 298-322 [en línea]. Brill. DOI: https://doi. org/10.1163/9789004428898_015

SCHOU, J.; HJELHOLT, M. (2018). Digitalization and public sector transformations [en línea]. Springer. DOI: https://doi.org/10.1007/978-3-319-76291-3

TROITIÑO, D. R.; KERIKMÄE, T.; CHOCHIA, A. (eds.) (2018). Brexit: History, reasoning and perspectives. Springer.

TROITIÑO, D. R.; KERIKMÄE, T.; DE LA GUARDIA, R. M.; SÁNCHEZ, G. Á. P. (eds.) (2020). The EU in the 21st Century: Challenges and Opportunities for the European Integration Process [en línea]. Springer Nature. DOI: https://doi.org/10.1007/978-3-030-38399-2

VAN NOORDT, C.; MISURACA, G. (2020). «Exploratory Insights on Artificial Intelligence for Government in Europe». En: Social Science Computer Review [en línea]. DOI: https://doi. org/10.1177/0894439320980449

ZEKOS, G. I. (2021). «E-Globalization and Digital Economy». En: Economics and Law of Artificial Intelligence, págs. 13-66 [en línea]. Cham: Springer. DOI: https://doi.org/10.1007/978-3-030-64254-9_2 


\title{
Cita recomendada
}

RAMIRO TROITIÑO, David (2021). «La "Década Digital" de la Unión Europea: Desarrollos e impactos sobre su ciudadanía y economía». IDP. Revista de Internet, Derecho y Política. Núm. 34. UOC [Fecha de consulta: dd/mm/aa] http://dx.doi.org/10.7238/idp.v0i34.387532

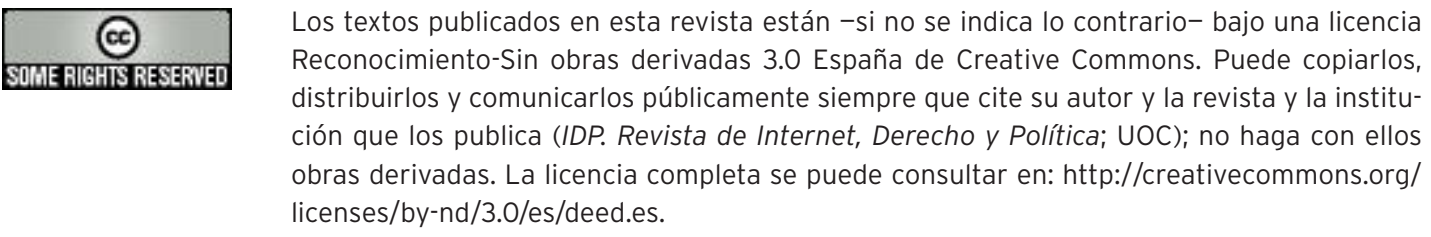

\author{
Sobre el autor \\ David Ramiro Troitiño \\ Universidad Tecnológica de Tallin (TalTech) \\ david.troitino@taltech.ee
}

Licenciado en Historia, Ciencias Políticas y Dirección y Administración de Empresas. Doctor en Historia Contemporánea en el programa de Estudios de la Unión Europea de la Universidad de Salamanca. Profesor titular de la Universidad Tecnológica de Tallin, Estonia, desde 2017. Anteriormente, docente en las universidades de Tartu, Concordia y Audentes, también localizadas en Estonia. Autor y coordinador de 5 libros académicos relacionados con la Unión Europea. Asimismo, autor o coautor de 122 artículos científicos publicados en revistas académicas internacionales indexadas. Líder de los siguientes proyectos europeos: Módulo Jean Monnet VERT672 «Functional Capacity of the European Union (1.09.2014-31.08.2017)»; VEU624 «WEBDATANET: web-based data-collection - methodological challenges, solutions and implementations (16.06.2011-15.06.2015)», David Ramiro Troitiño, Tallinn University of Technology, School of Business and Governance, Department of International Relations, Chair of European Studies; VEU20036 «Remember Nansen- migration, refugees and humanitarian aid (1.01.2021-30.06.2022)». Experto examinador de la Comisión Europea para la evaluación de proyectos. Conferenciante en Chile, Perú, Argentina, Colombia, España, Chipre, Turquía, Italia, Alemania, Letonia, Rusia, Malasia y Camboya.

ORCID: https://orcid.org/0000-0002-0542-5724

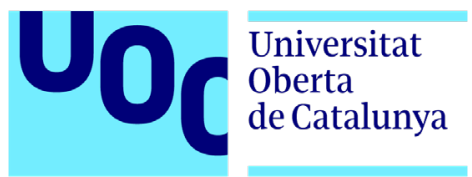

